\title{
RESPUESTA DE LA ACHICORIA (Cichorium intybus L.) A LA APLICACIÓN DE MAGNESIO
}

\section{CHICORY (Cichorium intybus L.) RESPONSE TO MAGNESIUM APPLICATION}

\author{
Susana Fischer ${ }^{1 *}$, Rosemarie Wilckens ${ }^{1}$, Iván Vidal ${ }^{1}$, Patricio Astete ${ }^{2}$, Johann Maier ${ }^{2}$ \\ ${ }^{1}$ Dep. Producción Vegetal, Facultad de Agronomía, Universidad de Concepción, Casilla 160-C, Chillán, \\ Chile. \\ ${ }^{2}$ Empresa Beneo, Departamento de Investigación, Ruta 5 Sur, Km 445 Fundo San Pedro 3, Pemuco, \\ Chillán, Chile. \\ *Autor para correspondencia E-mail: sfischer@udec.cl
}

\section{RESUMEN}

La achicoria (Cichorium intybus L.) es un cultivo de reciente introducción en Chile, para la producción de raíces que se cosechan el primer año de desarrollo para la obtención de inulina. El objetivo de este estudio fue evaluar el efecto de la aplicación de magnesio en tres dosis sobre el desarrollo de la achicoria, sintomatología foliar, concentración y absorción de nutrientes en hojas y raíz. El estudio se estableció en la Estación Experimental "El Nogal" de la Universidad de Concepción, empleando un diseño en bloques completo al azar con 5 repeticiones. La variedad utilizada fue Diesis, transplantadas con tres hojas verdaderas en bolsas de polietileno que contenían lana mineral. Durante el ensayo se aplicó una fertilización referencial mediante solución hidropónica (Solución Hoagland). Para la aplicación de magnesio se utilizaron tres concentraciones: deficitaria, referencial y tóxica. Durante el desarrollo del cultivo se evaluó la sintomatología y contenido de clorofila en hojas, peso fresco, peso seco y el análisis químico tanto de raíz como hoja en los estados de 7 y 14 hojas verdaderas, y a la cosecha. La sintomatología de las plantas con aplicación deficiente de magnesio fue marcada, observándose clorosis intervenal en hojas nuevas y crecimiento irregular de la lámina foliar. Las raíces alcanzaron entre el 60 al $67 \%$ del peso total de la planta. Las concentraciones de magnesio en hojas fueron mayores que en raíz, al igual que su absorción, la que aumentó hasta el desarrollo de 14 hojas verdaderas para posteriormente disminuir.

Palabras clave: sintomatología, concentración, absorción, macronutriente.

\section{ABSTRACT}

Chicory (Cichorium intybus L.) has been recently introduced in Chile as a rotational crop for the production of roots, which are harvested in the first year for inulin extraction. The objective of this study was to evaluate the effect of magnesium applied at three different doses on the growth of chicory, considering foliar symptoms, and concentration and absorption of nutrients in leaves and roots. The study was conducted at the 'El Nogal' Experimental Station of the University of Concepción, using a complete randomized block design with 5 replications. The variety used was Diesis, transplanted with three true leaves in polyethylene bags containing mineral wool. Reference fertilization was applied by hydroponic solution (Hoagland solution) during all plant development stages. Magnesium was applied in three different concentrations: deficient, reference and toxic. Measu- 
rements of symptomatology, chlorophyll content of leaves, fresh weight and dry matter weight were conducted in the stages 7 and 14 of true leaves and at harvest. Similarly, chemical analyses for both root and leaf were conducted at the same phenological stages. The symptoms for deficient application of magnesium were marked, showing chlorosis in new leaves and irregular growth of the leaf blade. The roots reached between 60 and $67 \%$ of total plant weight. Magnesium concentrations in leaves were higher in roots, as well as absorption, which increased until the development of the 14 true leaves and decreased towards harvest.

Key words: symptomatology, concentration, absorption, macronutrient.

\section{INTRODUCCIÓN}

La achicoria (Cichorium intybus L.) es una especie perteneciente a la familia Asteraceae, originaria de las zonas mediterráneas del norte de Europa (Vavilov, 1992). Es una herbácea bianual, en el primer año desarrolla una raíz profunda y gruesa, y hojas con peciolo corto, distribuidas en roseta. Posteriormente, una vez que la planta ha sido expuesta a temperaturas bajas, desarrolla un tallo vigoroso, en cuyo extremo se diferencian múltiples órganos reproductivos o flores (Demeulemeester y De Proft, 1999; Li and Kemp 2005).

En Chile esta especie es utilizada como cultivo industrial para producción de raíces. Al final del primer año de crecimiento y desarrollo, cuando la planta ha desarrollado una raíz madura, se extrae inulina y oligofructosas. Ambos carbohidratos, importantes en la industria alimentaria, son requeridos en la formulación de variados alimentos para aumentar la consistencia con un bajo aporte calórico, ofreciendo beneficios importantes a la salud (Van den Ende et al., 1996; Franck, 2006; Madrigal y Sangronis, 2007).

La producción de este cultivo ha debido adaptarse a las condiciones agroecológicas imperantes en Chile. Entre los factores importantes a considerar en el manejo, la fertilización cumple un rol preponderante en su productividad; en la actualidad se basa principalmente en macronutrientes, tales como nitrógeno, fósforo, potasio, además de la adición de algunos microelementos incorporados en las mezclas, como boro y zinc, entre otros (Orafti, 2015).

Tanto macro como micronutrientes son importantes para el crecimiento óptimo del cultivo, ya sea por integrar parte de una molécula o estar asociada al metabolismo general de la planta (Dominguez, 1989; Marschner, 2011). Las dosis requeridas de los microelementos son menores a los macro nutrientes. Sin embargo, tanto una toxicidad como una deficiencia de estos limita el ciclo de vida de la planta, provocando graves anormalidades en su crecimiento y desarrollo, lo que desde el punto de vista agronómico son perjudiciales para la producción (Rodríguez, 1996; Zobel et al., 2013).
Tanto la sobredosis como la carencia de nutrientes se manifiesta externamente en la planta a través de síntomas visuales característicos, aunque esta sintomatología puede confundirse en algunos casos con enfermedades $u$ otros daños fisiológicos, por lo que deben ser determinadas con métodos cuantitativos, tales como análisis de la planta entera o de alguna de sus parte, como hojas o raíz, y análisis de suelo (Vidal, 2007). Los efectos tóxicos son más recurrentes en macronutrientes, aunque igual pueden expresarse en casos extremos en microelementos.

Existen escasos antecedentes sobre el cultivo de la achicoria en Chile con respecto a las dosis de micronutrientes requerido a lo largo de su desarrollo y el efecto que estas producirían en el cultivo. Por lo anterior, el objetivo de este estudio fue evaluar el efecto de la aplicación de tres dosis de magnesio sobre el desarrollo, sintomatología foliar, concentración y absorción de nutrientes en hojas y raíz de la achicoria.

\section{MATERIALES Y MÉTODOS}

Ubicación del ensayo. Esta investigación se realizó en la Estación Experimental "El Nogal" $\left(36^{\circ} 35^{\prime} 43,2^{\prime \prime}\right.$ S, $72^{\circ} 04^{\prime} 39,9^{\prime \prime}$ O) de la Universidad de Concepción, Campus Chillán, Provincia de Ñuble, durante la temporada 2013-2014. El clima es mediterráneo templado con temperaturas anuales de $6,9^{\circ} \mathrm{C}$ como mínimo y $20,2^{\circ} \mathrm{C}$ como máximo, con un período libre de heladas de 5-6 meses y con una precipitación anual promedio de 1.200 mm (Del Pozo y Del Canto, 1999).

Material vegetal, establecimiento y manejo agronómico del experimento

En el experimento se utilizaron semillas de achicoria (Cichorium intybus L.) variedad Diesis; se sembraron en contenedores de 288 alveolos el 15 de noviembre 2013, y una vez que las plántulas alcanzaron tres hojas verdaderas, el 8 de diciembre de 2013, se trasplantaron 3 plantas por bolsas de polietileno negro, independientes, de $9 \mathrm{~cm}$ de radio por $50 \mathrm{~cm}$ de largo, expuestas a condiciones de campo, a la intemperie. Cada bolsa contenía lana mineral (Agrolan granulado, Volcan ${ }^{\circledR} 15 \mathrm{~kg}$ $\mathrm{m}^{-3}$ ) como sustrato inorgánico. Se aplicó la ferti- 
lización recomendada por Orafti (2015), con la misma dosis para todos los tratamientos, y una fertilización diferenciada con respecto a magnesio (Tabla 1). Durante el establecimiento del experimento se utilizaron tres concentraciones de magnesio: deficitaria $\left(0 \mathrm{mg} \mathrm{L}^{-1}\right)$, referencial $\left(30 \mathrm{mg} \mathrm{L}^{-1}\right)$ y tóxica (300 $\mathrm{mg} \mathrm{L}^{-1}$ ) (Tabla 1) la cual fue aplicada al sustrato en cada bolsa, utilizando la metodología sugerida para la fertirigación hidropónica (solución Hoagland) (Sonneveld and Voogt, 2009), con reactivos grado técnico (Merck®), con pureza mayor a 95\% (Tabla 2).

Los fertilizantes fueron suministrados de acuerdo a los protocolos sugeridos para fertiriego en sustrato inerte (Vidal, 2007), para lo cual se preparó una solución madre consistente en la disolución de distintas concentraciones de sales 100 veces concentrada diluida en $10 \mathrm{~L}$ de agua (Tabla 1), la cual al momento de realizar el fertirriego, se disolvió utilizando $495 \mathrm{~cm}^{3}$ de agua y $5 \mathrm{~cm}^{3}$ de la solución madre, aplicando a cada bolsa $500 \mathrm{~mL}$ de solución nutritiva a partir de los 15 días después de trasplante (DDT) hasta el desarrollo de 7 hojas verdaderas (H7), y posteriormente se aumentó a $1000 \mathrm{~cm}^{3}\left(990 \mathrm{~cm}^{3}\right.$ de agua y $10 \mathrm{~cm}^{3}$ de solución madre) hasta la cosecha $(\mathrm{C})$.

\section{Diseño experimental}

El diseño experimental establecido fue de bloques completamente al azar con 3 tratamientos y 5 repeticiones.

\section{Evaluaciones}

Caracterización de la sintomatología. Se realizó un análisis visual de todos los síntomas que presentaron las plantas a partir del segundo fertiriego [3 de enero 2014; 49 DDS (días después de siembra)] con una frecuencia de 15 días, determinando el número total de plantas que presentaron algún tipo de sintomatología visual (Bergmann, 1992).

Contenido de clorofila en hojas. Índice de clorofila. Se empleó un medidor de clorofila portátil (SPAD 502 Minolta). En cada unidad experimen-

Tabla 1. Concentración de magnesio y de otros nutrientes utilizados en cada tratamiento en experimento de achicoria (Cichorium intybus L.)

Table 1. Concentration of magnesium and other elements used per treatment in the chicory (Cichorium intybus L.) experiment.

\begin{tabular}{|c|c|c|c|c|c|c|c|c|c|c|c|c|}
\hline \multirow[t]{2}{*}{ Tratamiento } & \multicolumn{12}{|c|}{ Nutriente } \\
\hline & $\mathbf{N}$ & $\mathbf{P}$ & K & $S$ & $\mathrm{Ca}$ & Mg & $\mathrm{Zn}$ & Mn & B & $\mathrm{Cu}$ & Fe & Mo \\
\hline & --- & . &  & --. & ----- & $\mathrm{mg}$ & - & ---- & $\cdots$ & - & 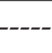 & --- \\
\hline Referencial $^{*}$ & 180 & 47 & 195 & 144 & 160 & 30 & 0,2 & 0,4 & 0,4 & 0,05 & 2 & 0,02 \\
\hline Deficitaria & 180 & 47 & 195 & 144 & 160 & 0 & 0,2 & 0,4 & 0,4 & 0,05 & 2 & 0,02 \\
\hline Tóxica & 180 & 47 & 195 & 144 & 160 & 300 & 0,2 & 0,4 & 0,4 & 0,05 & 2 & 0,02 \\
\hline
\end{tabular}

* Orafti, 2015.

Tabla 2. Elementos minerales y las respectivas fuentes empleadas para la nutrición de plantas de achicoria (Cichorium intybus L.)

Table 2. Mineral elements and commercial products used for nutrient supply in chicory (Cichorium intybus L.) plants.

\begin{tabular}{|c|c|}
\hline Elemento & Fuente empleada \\
\hline $\mathrm{N}$ & $\mathrm{KNO}_{3} ; \mathrm{NH}_{4} \mathrm{H}_{2} \mathrm{PO}_{4}$ \\
\hline $\mathrm{P}$ & $\mathrm{NH}_{4} \mathrm{H}_{2} \mathrm{PO}_{4}{ }_{4}^{2}$ \\
\hline K & $\mathrm{K}_{2} \mathrm{SO}_{4}^{4}$ \\
\hline$S$ & $\mathrm{~K}_{2}^{2} \mathrm{SO}_{4}^{4}$ \\
\hline $\mathrm{Ca}$ & $\mathrm{Ca}\left(\mathrm{NO}_{3}\right)_{2}$ \\
\hline $\mathrm{Mg}$ & $\mathrm{MgSO}_{4} \times 7 \mathrm{H}_{2} \mathrm{O}$ \\
\hline $\mathrm{Zn}$ & $\mathrm{ZnSO}_{4} \times \mathrm{H}_{2} \mathrm{O}$ \\
\hline $\mathrm{Mn}$ & $\mathrm{MnSO}_{4}^{4} \times 2 \mathrm{H}_{2} \mathrm{O}$ \\
\hline B & $\mathrm{H}_{3} \mathrm{BO}_{4}{ }^{4}$ \\
\hline $\mathrm{Cu}$ & $\mathrm{CuSO}_{4}^{3} \times 5 \mathrm{H}_{2} \mathrm{O}$ \\
\hline $\mathrm{Fe}$ & EDTA Fe \\
\hline Mo & $\mathrm{Na}_{2} \mathrm{MoO}_{4} \times 2 \mathrm{H}_{2} \mathrm{O}$ \\
\hline
\end{tabular}


tal se efectuaron 3 mediciones al azar en hojas del tercio superior de la planta y a la misma hora.

Peso fresco y seco biomasa. Se evaluó la producción de materia fresca y seca de raíz y de hojas en diferentes estados fenológicos: planta con 7 hojas verdaderas (H7) (21 de enero 2014; 67 DDS), 14 hojas verdaderas (H14) (18 marzo 2014, 122 DDS) y a la cosecha (C) (10 de abril 2014; 145 DDS). Las muestras fueron secadas en una estufa a $80^{\circ} \mathrm{C}$ por un periodo de $60 \mathrm{~h}$. Los resultados se expresaron en $\mathrm{g} \mathrm{pl}^{-1}$.

Análisis químico. Para cada fracción vegetal, hoja o raíz, se realizó un análisis químico en cada muestreo (H7, H14 y C), determinando en el tratamiento de referencia la absorción de $\mathrm{N}, \mathrm{P}, \mathrm{K}, \mathrm{Fe}$, $\mathrm{Ca}, \mathrm{B}, \mathrm{Mn}$ y Mg (Tabla 3). Por otra parte, se determinó la absorción y concentración de magnesio en los tratamientos tóxico y deficiente.

Las digestiones de $\mathrm{N}, \mathrm{P}, \mathrm{K}, \mathrm{Ca}, \mathrm{Mn}, \mathrm{y} \mathrm{Mg}$, se realizaron en muestras de hoja y raíz seca y molida a $>1 \mathrm{~mm}$. Cada una por separado fueron digeridas en un matraz con ácido sulfúrico, ácido salicílico y agua oxigenada. Para la digestión de B y Fe se tomaron muestras de $1 \mathrm{~g}$ de cada tejido, las que fueron secadas, molidas a $>1 \mathrm{~mm}$ y calcinadas a $500^{\circ} \mathrm{C}$ y las cenizas disueltas en ácido clorhídrico diluido (Sadzawka et al., 2007). La determinación de $\mathrm{N}$ se hizo por destilación y titulación manual, del $\mathrm{P}$ por colorimetría con nitro-vanado-molibdato, la determinación de $\mathrm{K}, \mathrm{Fe}, \mathrm{Ca}$, Mn y Mg el por espectrofotometría de absorción atómica (EAA) con llama de aire-acetilento por aspiración directa. El B fue determinado por colorimetría con azometina-H (Sadzawka et al., 2007).

\section{Análisis de datos}

Los datos obtenidos fueron sometidos a análisis de varianza, previa comprobación de los supuestos de dicho análisis, y sus medias fueron comparadas mediante el test DMS $(\mathrm{P} \leq 0,05)$. Para realizar todos los análisis se empleó el paquete estadístico SAS (2003).

\section{RESULTADOS Y DISCUSIÓN}

\section{Caracterización de la sintomatología.}

A los 51 DDS se manifestó la sintomatología de deficiencia, observándose clorosis intervenal en hojas nuevas (Figura 1A) y un leve crecimiento irregular (encarrujado) de la lámina foliar. Esta tendencia se mantuvo en el tratamiento de deficiencia agudizándose hacia la cosecha, evidenciándose un menor crecimiento y clorosis generalizada en hojas adultas, y pardeamiento en el ápice de la lámina (Figura 1B). En el tratamiento referencial y tóxico no se observaron sintomatologías.

La sintomatología de clorosis por deficiencia de $\mathrm{Mg}$ se explica debido a que el ión se ubica al centro del tetrapirrol de la molécula de clorofila, constituyendo entre el 6 a 35\% del Mg total de la planta, dependiendo de su estado de desarrollo (Scott y Robson, 1990). Desde el punto de vista fisiológico, el Mg, que es un elemento móvil dentro de la planta y requerido para sintetizar la molécula de clorofila, al no estar disponible en cantidades suficientes en la rizosfera, es traslocado desde otra parte de la planta, vía floema, hacia los puntos de crecimiento activo, como las hojas nuevas (Cakmak y Kirkby, 2008). Por otra parte, según Merhaut (2007) en algunas plantas es un síntoma

Tabla 3. Absorción de macro elementos, fierro, manganeso y boro en hojas y raíz de achicoria (Cichorium intybus L.) en distintos estados fenológicos para el tratamiento referencial.

Table 3. Absorption of macro elements, iron, manganese and boron in chicory (Cichorium intybus L.) leaves and roots at different growth stages in the reference treatment.

\begin{tabular}{|c|c|c|c|c|c|c|c|c|}
\hline \multicolumn{2}{|c|}{$\begin{array}{l}\text { Estado } \\
\text { desarrollo }\end{array}$} & $\mathbf{P}_{2} \mathbf{O}_{5}$ & $\mathrm{~K}_{2} \mathrm{O}$ & $\mathrm{Ca}$ & $\mathrm{Mg}$ & $\mathrm{Fe}$ & Mn & B \\
\hline & \multicolumn{4}{|c|}{ 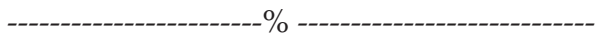 } & \multicolumn{4}{|c|}{ - } \\
\hline \multicolumn{9}{|l|}{ Hojas } \\
\hline $\mathrm{H} 7$ & $2,8 \pm 0,5$ & $0,2 \pm 0,01$ & $5,1 \pm 0,5$ & $1,3 \pm 0,2$ & $0,3 \pm 0,02$ & $94 \pm 15$ & $69,5 \pm 7,6$ & $21,9 \pm 3,7$ \\
\hline H14 & $2,1 \pm 0,1$ & $0,3 \pm 0,10$ & $4,1 \pm 0,1$ & $2,0 \pm 0,6$ & $2,8 \pm 0,45$ & $191 \pm 21$ & $100 \pm 10,3$ & $30,7 \pm 2,5$ \\
\hline $\mathrm{C}$ & $1,7 \pm 0,2$ & $0,2 \pm 0,06$ & $3,4 \pm 0,1$ & $2,4 \pm 0,2$ & $0,3 \pm 0,04$ & $103 \pm 22$ & $111 \pm 26,7$ & $57,5 \pm 4,7$ \\
\hline \multicolumn{9}{|l|}{ Raíz } \\
\hline $\mathrm{H} 7$ & $1,1 \pm 0,3$ & $0,1 \pm 0,03$ & $1,7 \pm 0,4$ & $1,9 \pm 0,2$ & $0,1 \pm 0,05$ & $97 \pm 15.1$ & $20,7 \pm 3,4$ & $25,4 \pm 5,7$ \\
\hline H14 & $1,5 \pm 0,6$ & $0,2 \pm 0,03$ & $1,7 \pm 0,1$ & $1,6 \pm 0,1$ & $0,1 \pm 0,05$ & $190 \pm 6.4$ & $22,0 \pm 6,0$ & $10,0 \pm 2,6$ \\
\hline $\mathrm{C}$ & $1,3 \pm 0,1$ & $0,2 \pm 0,02$ & $1,7 \pm 0,9$ & $1,5 \pm 0,2$ & $0,1 \pm 0,03$ & $69 \pm 8,2$ & $21,0 \pm 1,8$ & $17,9 \pm 8,8$ \\
\hline
\end{tabular}

H7 = siete hojas verdaderas; H14 = catorce hojas verdaderas; C = cosecha; \pm = desviación estándar. 


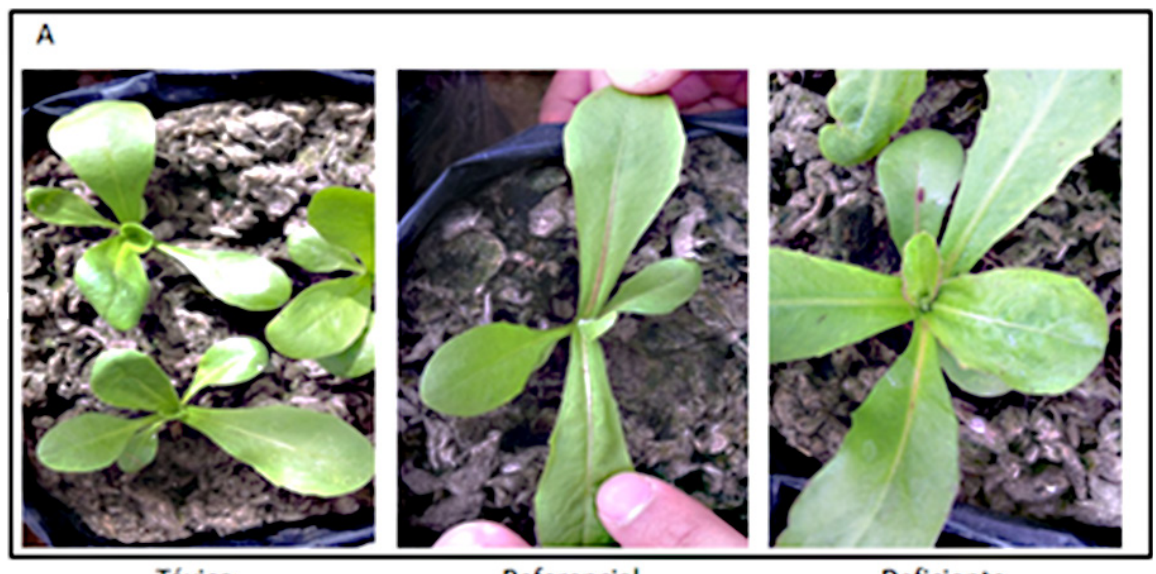

Tóxico

Referencial

Deficiente

B

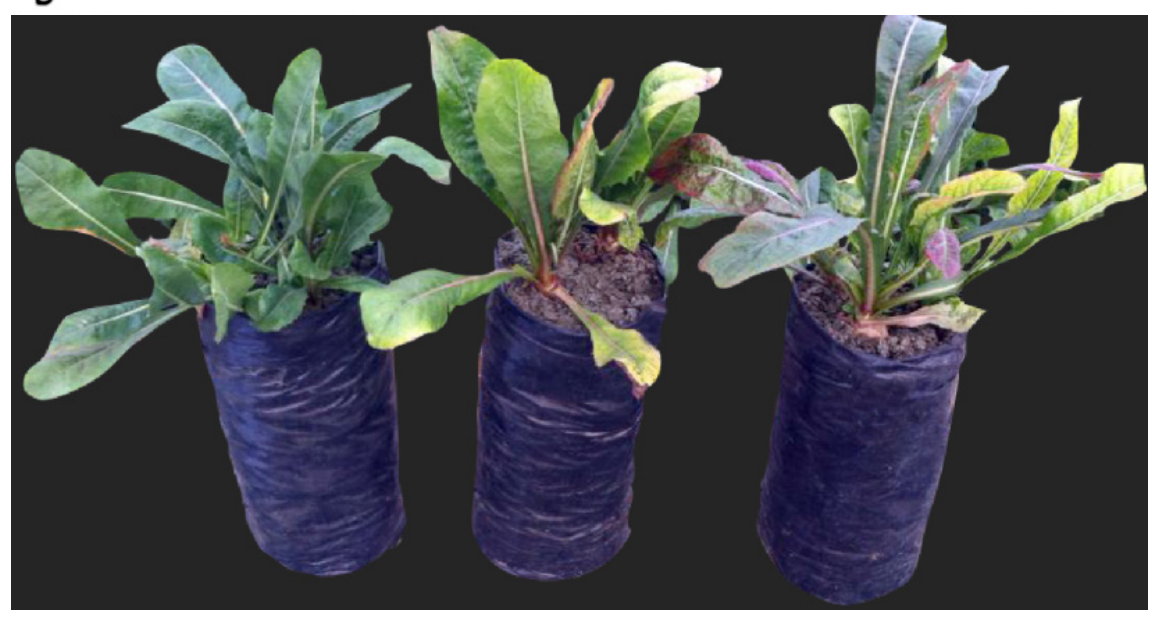

Fig. 1. Efecto del magnesio en hojas de achicoria (Cichorium intybus L.). A) A los 51 días después de la siembra; B) A la cosecha, 145 días después de la siembra.

En la foto de izquierda a derecha: tratamientos tóxicos, referencial y deficitarios, respectivamente.

Fig. 1. Effect of magnesium on chicory (Cichorium intybus L.) leaves. A) 51 days after sowing; B) At harvest, 145 days after sowing.

In the picture from left to right: toxic, reference and deficient treatments, respectively.

característico de esta deficiencia el posterior pardeamiento de las hojas

\section{Índice de clorofila}

El índice de clorofila observado está relacionado con la sintomatología anteriormente descrita, ya que los tratamientos de referencia y de toxicidad presentaron una tendencia similar en las etapas de $\mathrm{H7}$ y H14, con diferencias significativas ( $\mathrm{P}$ $\leq 0,05$; Tabla 4). Se observó que la concentración de clorofila aumentó a medida que la planta se desarrolló hasta el estado de 14 hojas verdaderas (122 DDS), disminuyendo hacia la cosecha (145 DDS) a valores SPAD de 42,8 y 52,5, respectivamente, con valores mayores en el tratamiento tó- xico respecto al referencial. Sin embargo, para el tratamiento deficitario el valor disminuyó hasta los 122 DDS, manteniéndose sin variación hasta la cosecha (Tabla 4). Dado que el 15 al 30\% del total de magnesio de la planta está asociado a la molécula de clorofila, una deficiencia de este elemento se asocia directamente con una disminución de la concentración de clorofila en las hojas (Lavon y Goldsmidt, 1999; White y Brown 2010).

Por otra parte, se ha observado que la deficiencia de $\mathrm{Mg}$ puede causar deformación de las células floemáticas y el colapso de las células cribosas, alteraciones que ocurren antes del cambio visual evidente de clorosis en hojas y una reducción en la tasa neta de fotosíntesis (Palomäki, 
Tabla 4. Lecturas SPAD de clorofila en hojas de achicoria (Cichorium intybus L.) en tratamientos con distintas concentraciones de magnesio.

Table 4. SPAD chlorophyll readings from chicory (Cichorium intybus L.) leaves under different concentrations of magnesium.

\begin{tabular}{|c|c|c|c|}
\hline \multirow{2}{*}{$\begin{array}{l}\text { Tratamiento } \\
\text { Magnesio }\end{array}$} & \multicolumn{3}{|c|}{ Estado desarrollo } \\
\hline & H7 & H14 & Cosecha \\
\hline & - & Clorofila (\%) &  \\
\hline Deficiente & $44,6 \mathrm{~b} \pm 1,5$ & $38,0 \mathrm{~b} \pm 1,0$ & $38,4 \mathrm{a} \pm 2,9$ \\
\hline Referencia & $46,7 \mathrm{~b} \pm 1,1$ & $47,6 \mathrm{~b} \pm 4,7$ & $42,8 \mathrm{a} \pm 1,4$ \\
\hline Tóxico & $51,7 \mathrm{a} \pm 3,0$ & $54,0 \mathrm{a} \pm 2,7$ & $52,5 \mathrm{a} \pm 4,4$ \\
\hline DMS $(0,05)$ & 4,1 & 5,8 & 9,7 \\
\hline
\end{tabular}

H7 = siete hojas verdaderas; $\mathrm{H} 14$ = catorce hojas verdaderas; $\mathrm{C}=$ cosecha; $\pm=$ desviación estándar.

1995; Farhat et al., 2014). En relación a la respuesta de las plantas sometidas a la condición de toxicidad de $\mathrm{Mg}$, resulta contradictorio que en sus hojas haya aumentado la cantidad de clorofila, ya que autores como Chutia y Prasad (2012) señalan que la condición de stress dada por la toxicidad disminuye la cantidad de clorofila a y b en hojas. Una posible explicación, para los niveles de $\mathrm{Mg}$ observados en este ensayo, podría sustentarse en el secuestro del magnesio presente en exceso, por acción de la vacuola, regulado por el calcio del tonoplasto, la proteína CBL (calcineurin B-like protein) y la CIPKs (CBL-interacting protein kinases) (Gao et al., 2015).

\section{Peso fresco y seco de biomasa}

En relación al desarrollo de hojas, durante los 122 DDS aumentó el peso fresco y seco hasta cuando la planta desarrolló 14 hojas verdaderas. Sin embargo, posteriormente hay una inflexión, en la cual disminuyen tanto el peso fresco como el peso seco hasta la cosecha, realizada a los 145 DDS (Tabla 5). Este comportamiento se podría atribuir al desarrollo fenológico propio de esta especie, y otras bianuales, en las cuales se deprime el crecimiento de hojas cuando la temperatura ambiental desciende (Devacht et al., 2009). Por otra parte, las hojas al madurar entran en senescencia y en consecuencia hay una pérdida de biomasa. Se ha observado una menor tasa de crecimiento y adelanto en la senescencia de hojas en plantas expuestas a deficiencias de $\mathrm{Mg}$, asociado a un desbalance en la concentración de azúcares, principalmente en hojas, y a la muerte de tejido causado por la acumulación de especies reactivas de oxígeno, producto del estrés por la deficiencia (Hermans et al., 2004; Tewari et al., 2006).

El peso fresco y seco de las hojas en todos los tratamientos presentó diferencia significativa $(\mathrm{P} \leq$ $0,05)$ a los 122 y 145 DDS, respectivamente (Tabla 5). En las plantas del tratamiento referencial, en todas las fechas de muestreo, los valores de ambos parámetros fueron mayores que los tratamientos tóxico y deficitario (Tabla 5). Igual comportamiento se observó en raíces, alcanzando el tratamiento referencial un peso seco de $10,3 \mathrm{~g} \mathrm{pl}^{-1}$ a la cosecha, siendo estadísticamente diferente $(\mathrm{P}$ $\leq 0,05$ ) al tratamiento deficitario, cuyo rendimiento fue $6 \mathrm{~g} \mathrm{pl}^{-1}$, respectivamente (Tabla 5).

Con respecto al crecimiento de raíz de achicoria, a la cosecha alcanzó alrededor del $60 \%$ del peso total de la planta en el tratamiento de referencia y deficiente, mientras que en el tratamiento tóxico fue de un $67 \%$ (Tabla 5). Este rendimiento de raíces fue inferior al rendimiento comercial promedio en la zona de cultivo en Chile (ODEPA, 2015). Al proyectar el rendimiento comercial usando el peso de raíz determinado en este ensayo, y considerando una densidad de 177.000 plantas ha-1 ${ }^{-1}$ se obtendría sólo 9,1 t ha ${ }^{-1}$. Esta diferencia de rendimiento se podría atribuir al reducido volumen de las macetas empleadas, que no permitieron el desarrollo normal del sistema radicular (NeSmith y Duval, 1998; Poorter et al., 2012).

\section{Análisis químico}

Se observó que en plantas tratadas con dosis tóxica de $\mathrm{Mg}$, su concentración fue mayor en ambos órganos durante todas las etapas fenológicas del cultivo respecto a los otros dos tratamientos (Tabla 6). Por otra parte, las concentraciones de $\mathrm{Mg}$ radicular comparadas con la foliar fueron menor en todas las evaluaciones, independiente del tratamiento aplicado.

Con respecto a las dosis aplicadas, se observó una disminución de la concentración de $\mathrm{Mg}$ en hojas del tratamiento referencial, mientras que en raíces este tratamiento permaneció relativamente constante. En plantas fertirigadas con dosis referencial se observó diferencias estadísticas ( $\mathrm{P} \leq$ 0,05), en las raíces, a partir de los 122 DDS (H14) 
Tabla 5. Efecto de la suplementación de magnesio sobre la producción de biomasa en los diferentes órganos de achicoria (Cichorium intybus L.).

Table 5. Effect of magnesium supplementation on biomass production in different chicory (Cichorium intybus L.) organs.

\begin{tabular}{|c|c|c|c|c|c|c|}
\hline \multirow[t]{2}{*}{ Magnesio } & \multicolumn{3}{|c|}{ Peso fresco } & \multicolumn{3}{|c|}{ Peso seco } \\
\hline & H7 & H14 & Cosecha & H7 & H14 & Cosecha \\
\hline & -------------. & --------------- & --- $\mathrm{g} \mathrm{pl}^{-1}$ & & 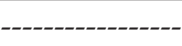 & ------------- \\
\hline \multicolumn{7}{|l|}{ Hojas } \\
\hline Deficiente & $9,0 \mathrm{a} \pm 0,7$ & $26,0 \mathrm{~b} \pm 4,1$ & $17,5 \mathrm{a} \pm 3,8$ & $1,2 \mathrm{a} \pm 0,1$ & $5,7 \mathrm{a} \pm 1,2$ & $3,7 b \pm 0,9$ \\
\hline Referencia & $7,6 \mathrm{a} \pm 1,5$ & $43,0 \mathrm{a} \pm 10,5$ & $29,8 \mathrm{a} \pm 5,2$ & $1,1 \mathrm{a} \pm 0,2$ & $8,8 \mathrm{a} \pm 3,1$ & $7,0 \mathrm{a} \pm 1,7$ \\
\hline Tóxico & $7,3 \mathrm{a} \pm 1,2$ & $24,4 \mathrm{a} \pm 11,2$ & $21,6 \mathrm{a} \pm 5,2$ & $1,0 \mathrm{a} \pm 0,2$ & $4,5 \mathrm{a} \pm 2,2$ & $4,3 b \pm 4,3$ \\
\hline DMS $(0,05)$ & 2,37 & 13,2 & 9,58 & 3,92 & 0,37 & 2,34 \\
\hline \multicolumn{7}{|l|}{ Raíz } \\
\hline Deficiente & --- & $39,0 b \pm 6,2$ & $33,6 b \pm 6,4$ & --- & $8,4 b \pm 1,9$ & $6,0 b \pm 0,4$ \\
\hline Referencia & $2,1 \pm 0,6$ & $64,4 \mathrm{a} \pm 15,8$ & $51,6 \mathrm{a} \pm 1,2$ & $0,3 \pm 0,1$ & $15,9 \mathrm{a} \pm 4,6$ & $10,3 \mathrm{a} \pm 0,8$ \\
\hline Tóxico & --- & $29,9 b \pm 7,6$ & $40,1 b \pm 10,2$ & --- & $7,2 \mathrm{~b} \pm 1,7$ & $8,7 \mathrm{a} \pm 2,2$ \\
\hline DMS $(0,05)$ & --- & 21,5 & 14,0 & --- & 6,1 & 2,7 \\
\hline
\end{tabular}

Letras distintas en la misma columna indican que los valores difieren significativamente.

hasta la cosecha, siendo los valores superiores a los tratamientos tóxico y deficitario (Tabla 6). En el tratamiento tóxico y deficitario se observó un comportamiento similar, en hojas donde la concentración de $\mathrm{Mg}$ disminuyó durante el desarrollo fenológico de la planta, sin embargo en raíces este comportamiento se observó sólo en el tratamiento tóxico, o sin alcanzar valores sobre el $1 \%$, en ambos tejidos (Tabla 6).

La absorción de $\mathrm{Mg}$ en hojas de achicoria fue mucho mayor que en raíz, la cual alcanza niveles no mayores a $13,7 \mathrm{mg} \mathrm{pl}^{-1}$ a la cosecha (Tabla 6). Al respecto, los niveles de $\mathrm{Mg}$ en hojas aumentaron hasta los 122 DDS, independiente del tratamiento, y posteriormente disminuyeron hasta la cosecha, siendo significativamente diferente con respecto a los dos tratamientos restantes $(\mathrm{P} \leq 0,05$; Tabla 6). $\mathrm{Al}$ respecto se ha demostrado que la retraslocación de $\mathrm{Mg}$ desde las hojas maduras a las nuevas, es lenta (Hermans et al., 2013) y justamente después de los 122 DDS se observó una pérdida paulatina de hojas maduras, que senecieron por

Tabla 6. Efecto de la suplementación de magnesio sobre la concentración y absorción de magnesio sobre diferentes órganos en achicoria (Cichorium intybus L.).

Table 6. Effect of magnesium supplementation on the concentration and absorption of magnesium by different organs in chicory (Cichorium intybus L.).

\begin{tabular}{|c|c|c|c|c|c|c|}
\hline \multirow{2}{*}{$\begin{array}{l}\text { Tratamiento } \\
\text { Magnesio }\end{array}$} & \multicolumn{3}{|c|}{ Concentración } & \multicolumn{3}{|c|}{ Absorción } \\
\hline & H7 & H14 & Cosecha & H7 & H14 & Cosecha \\
\hline H. & ---- & ---- \% & --- & --- & $\mathrm{mg} \mathrm{pl}^{-1}$ & -------- \\
\hline Deficiente & $0.17 c+0.02$ & $0,14 c+0,09$ & $0.13 b+0.01$ & $2,02 b+0.17$ & $8,09 b+3,22$ & $4,88 \mathrm{~b} \pm 1.1$ \\
\hline Referencia & $0,30 \mathrm{~b} \pm 0,02$ & $0,29 a \pm 0,01$ & $0,27 \mathrm{a} \pm 0,01$ & $3,33 b \pm 0,52$ & $25,4 \mathrm{a} \pm 1,14$ & $19,30 \mathrm{a} \pm 3,26$ \\
\hline Tóxico & $0,69 c \pm 0,05$ & $0,23 b \pm 0,02$ & $0,18 b \pm 0,17$ & $6,84 \mathrm{a} \pm 1,5$ & $16,1 \mathrm{ab} \pm 4,30$ & $7,50 b \pm 1,08$ \\
\hline DMS $(0,05)$ & 0,07 & 0,04 & 0,05 & 1,87 & 10,55 & 8,60 \\
\hline \multicolumn{7}{|l|}{ Raíz } \\
\hline Deficiente & --- & $0,11 b \pm 0,01$ & $0,06 \mathrm{~b} \pm 0,03$ & --- & $8,95 b \pm 1,24$ & $3,80 b \pm 1,99$ \\
\hline Referencia & $0,13 \pm 0,05$ & $0,11 b \pm 0,01$ & $0,11 \mathrm{ab} \pm 0,03$ & $0,40 \pm 0,1$ & $18,1 \mathrm{a} \pm 1,24$ & $11,7 \mathrm{a} \pm 2,66$ \\
\hline Tóxico & --- & $0,22 a \pm 0,02$ & $0,16 a \pm 0,05$ & --- & $15,9 a b \pm 3,39$ & $12,9 \mathrm{a} \pm 1,81$ \\
\hline DMS $(0,05)$ & --- & 0,03 & 0,07 & --- & 7,34 & 4,4 \\
\hline
\end{tabular}

$\pm=$ desviación estándar. 
efecto de la disminución de las temperaturas ambientales propias del otoño en la zona donde se realizó el estudio. En raíces el contenido de $\mathrm{Mg}$ desde 122 DDS a la cosecha en el tratamiento de referencia alcanzó valores de $11,7 \mathrm{mg} \mathrm{pl}^{-1}$, similares a los tóxicos (Tabla 6).

El $\mathrm{Mg}$ está directamente involucrado en los procesos primarios de la fotosíntesis, siendo esencial para la síntesis y mantención de la clorofila y las proteínas en los tilacoides de los cloroplastos (Papenbroock et al., 2000). Según los resultados, la distribución del magnesio fue diferente entre los distintos órganos de la planta, encontrándose en mayor cantidad en órganos que transpiran, tal como hojas y flores, y en menor medida en las raíces (Drossopoulos et al., 1996; Courtois et al., 2003; Ríos et al., 2012). Además, se ha descrito que la concentración de Mg varía de acuerdo al estado fenológico en que se encuentra la planta (Wilkinson et al., 1990; Marschner, 2011).

Las concentraciones de magnesio determinadas en achicoria en la presente investigación están dentro de los parámetros descritos (Merhaut, 2007; White y Brown, 2010). Disponer de información con respecto a la curva de acumulación de los elementos minerales en el cultivo a lo largo de su desarrollo permite planificar una intervención adecuada con respecto a la aplicación de fertilizantes. Al respecto, la concentración de magnesio varió entre el 0,1 y 0,5\% del peso seco. Sin embargo, estos valores pueden variar considerablemente entre las especies y las familias botánicas (Marschner, 2011)

\section{CONCLUSIONES}

Las plantas de achicoria que no fueron fertirrigadas con magnesio presentaron sintomatología de deficiencia asociada a clorosis generalizada en hojas adultas y posterior pardeamiento. Tanto hojas como raíz de plantas tratadas con dosis referencial de magnesio alcanzaron los mayores pesos frescos y secos durante todo el desarrollo del cultivo. La concentración de magnesio fue mayor en plantas expuestas a una dosis tóxica de este elemento, con un mayor porcentaje en hojas. La absorción de magnesio aumentó hasta el desarrollo de 14 hojas verdaderas para disminuir posteriormente.

\section{LITERATURA CITADA}

Bergmann, W. 1992. Nutritional disorders of plants. Development, visual and analytical diagnosis. Gustav Fischer, Jena, Germany.

Cakmak, I., and E.A. Kirkby. 2008. Role of magnesium in carbon partitioning and alleviating photooxidative damage. Physiologia Plantarum 133(4):692-704.

Courtoisa, D., P. Kastenmayerb, J. Cloughb, M. Vigob, M. Sabatierc, and M. J. Arnaudc. 2003. Magnesium enrichment and distribution in plants. Isotopers in environmental and health studies 39(4):273-279.

Chutia, J. and S. Prasad. 2012. A water stress effects on leaf growth and chlorophyll content but not the grain yield in traditional rice (Oryza sativa Linn.) genotypes of Assam, India II. Protein and proline status in seedlings under PEG induced water stress. American Journal of Plant Sciences 3:971-980.

Del Pozo, A., y P. Del Canto. 1999. Áreas agroclimáticas y sistemas productivos en la VII y VIII Regiones. Serie Quilamapu Nº 113. INIA Quilamapu, Chillán, Chile.

Demeulemeester, M.A.C, and M.P. De Proft. 1999. In vivo and in vitro flowering response of chicory (Cichorium intybus L.): influence of plant age and vernalization. Plant Cell Reports 18:781-785.

Devacht, S., P. Lootens, I. Roldán-Ruiz, L. Carlier, J. Baert, J. Van Waes, and E. Van Bockstaele. 2009. Influence of low temperatures on the growth and photosynthetic activity of industrial chicory, Cichorium intybus L. Photosynthetica 47(3):372-380.

Dominguez, A. 1989. Tratado de fertilización. 3a. ed. Mundi Prensa, Madrid, España.

Drossopoulos, B., G.G. Kouchaji, and D.L. Bouranis. 1996. Seasonal dynamics of mineral nutrients and carbohydrates by walnut tree leaves. Journal of Plant Nutrition 19(3-4):493-516.

Farhat, N., M. Rabhi, M. Krol, Z., Barhoumi, A.G. Ivanov, A. McCarthy, and N.P. Hüner. 2014. Starch and sugar accumulation in Sulla carnosa leaves upon $\mathrm{Mg}^{2+}$ starvation. Acta Physiologiae Plantarum 36(8):2157-2165.

Franck, A. 2006. Inulin. p. 335-349. In A. Stephen, G. Phillips and P. Williams (eds.). Food polysaccharides and their applications. 2nd. ed. CRS Press, New York, USA.

Gao, C., Q. Zhao, and L. Jiang. 2015. Vacuoles protect plants from high magnesium stress. Proceedings of the National Academy of Sciences of the United States of America 112(10):2931-2932. Available at http://doi. org/10.1073/pnas.1501318112. (Accessed 5 January 2016). 
Hermans, C., G.N. Johnson, R.J. Strasser, and N. Verbruggen. 2004. Physiological characterization of magnesium deficiency in sugar beet: acclimation to low magnesium differentially affects photosystems I and II. Planta 220(2):344-355.

Hermans, C., S.J. Conn, J. Chen, Q. Xiao, and N. Verbruggen. 2013 An update on magnesium homeostasis mechanisms in plants. Metallomics 5:1170-1183.

Lavon R., R. Salomon, and E.E. Goldschmidt. 1999. Effect of potassium, magnesium, and calcium deficiencies on nitrogen constituents and chloroplast components in citrus leaves. J. Am. Soc. Hort. Sci. 124:158-162.

Li, G., and P.D. Kemp. 2005. Forage chicory (Cichorium intybus L.): A review of its agronomy and animal production. Advances in Agronomy 88:187-222.

Madrigal, L., y E. Sangronis. 2007. La inulina y derivados como ingredientes claves en alimentos funcionales. Archivos Latinoamericanos de Nutrición 57(4):387-395.

Marschner, P. 2011. Marschner's mineral nutrition of higher plants. 3th. ed. Academic Press, San Diego, USA.

Merhaut, D.J. 2007. Magnesium. p. 146-181. In A.V. Backer and D.J. Pilbeam (eds.). Handbook of plants nutrition. CRC Press, Boca Raton, Florida. USA.

NeSmith, D., and J.R. Duval. 1998. The effect of container size. HortTechnology 8(4):495-498.

ODEPA. 2015. Oficina de Estudios y Políticas Agrarias (ODEPA), Santiago, Chile. Disponible en http://www.odepa.cl/cultivos-anuales-superficie-produccion-y-rendimientos-4/ (Consulta 20 marzo 2015).

Orafti. 2015. Manual técnico de cultivo. Disponible en http://www.orafti.cl/download/manualtecnicocultivo2014-2015.pdf (Consulta 20 marzo 2015).

Palomäki, V. 1995. Effects of magnesium deficiency on needle ultraestructure and growth of Scots pine seedlings. Can. J. Forest Res. 25(11):1806-1814.

Papenbrock, J., E. Pfündel, H.P. Mock, and B. Grimm. 2000. Decreased and increased expression of the subunit CHL I diminishes $\mathrm{Mg}$ chelatase activity and reduces chlorophyll synthesis in transgenic tobacco plants. The Plant Journal 22(2):155-164.

Poorter, H., J. Bühler, D. van Dusschoten, J. Climent, and J.A. Postma. 2012. Pot size matters: a meta-analysis of the effects of rooting volume on plant growth. Functional Plant Biology 39(11): 839-850.
Rios, J.J., S.O Lochlainn, J. Devonshire, N.S. Graham, J.P. Hammond, G. J. King, et al. 2012. Distribution of calcium (Ca) and magnesium $(\mathrm{Mg})$ in the leaves of Brassica rapa under varying exogenous $\mathrm{Ca}$ and $\mathrm{Mg}$ supply. Annals of Botany 109:1081-1089.

Rodríguez, F. 1996. Fertilizantes: nutrición vegetal. A.G.T Editor, DF, México.

Sadzawka, A., M.A. Carrasco, R. Demantet, H. Flores, R. Grez, M. Mora, y A. Neaman. 2007. Métodos de análisis de tejidos vegetales. $\mathrm{p}$. 61-109. Serie Actas INIA N ${ }^{\circ}$ 40. INIA La Platina, Santiago, Chile.

SAS. 2003. SAS User's Guide. Statistic. SAS Institute Inc., Cary, North Caroline, USA.

Scott B., and A. Robson. 1990. Changes in the content and form of magnesium in the first trifoliate leaf of subterranean clover under altered or constant root supply. Aust. J. Agric. Res. 41:511-519.

Sonneveld, C., and W. Voogt. 2009. Plant nutrition of greenhouse crops. 431 p. Springer Science Business Media, Dordrecht, Wageningen, Netherland.

Tewari, R.K., P. Kumar, and P.N. Sharma. 2006. Magnesium deficiency induced oxidative stress and antioxidant responses in mulberry plants. Scientia Horticulturae 108(1):7-14.

Van den Ende, W., A. Mintiens, H. Speleers, A. Onuoha, and A. Laere. 1996. The metabolism of fructans in roots of Cichorium intybus during growth, storage and forcing. New Phytologist 132(4):555-563.

Vavilov, N.I. 1992. Origin and geography of cultivated plants. In V.F. Dorofeyev (ed.). Cambridge University Press, Cambridge, Great Britain.

Vidal, I. 2007. Fertirrigación cultivos y frutales. Facultad de Agronomía, Universidad de Concepción, Chillán, Chile.

White, P.J., and P.H. Brown. 2010. Plant nutrition for sustainable development and global health. Annals of Botany 105:1073-1080.

Wilkinson, S.R., R.M. Welch, H.F. Mayland, and D.L. Grunes. 1990. Magnesium in plants: uptake, distribution, function, and utilization by man and animals. Annals of Botany 105:1073-1080.

Zobel, R.W, K.A. Cassidaa, T.B. Kinraidea, J.M. Gonzalez, and J.G. Foster. 2013. Morphological response of forage chicory fine roots to manipulated soil phosphorus levels. Journal of Plant Nutrition 36:1901-1914. 